\title{
Angular correlation of electrons and positrons in internal pair conversion
}

\author{
Ch. Hofmann, J. Reinhardt, and W. Greiner \\ Institut für Theoretische Physik der J. W. Goethe Universität, Postfach 111 932, \\ D-6000 Frankfurt am Main 11, Germany \\ P. Schlüter \\ Siemens Aktiengesellschaft, Zentralabteilung Forschung und Entwicklung, Postfach 830 953, \\ D-8000 München 83, Germany \\ G. Soff \\ Gesellschaft für Schwerionenforschung, Planckstrasse 1, Postfach 110 552, D-6100 Darmstadt, Germany
}

(Received 2 July 1990)

\begin{abstract}
The angular distribution of electrons and positrons emitted in internal pair conversion is calculated. Coulomb-distorted waves are used as electron wave functions. Nuclear transitions of various multipolarities $L>0$ and of magnetic (ML) and of electric (EL) type are considered as well as $E 0$ conversion. Analytical expressions for the angular correlation are derived, which are evaluated numerically assuming a finite extension of the nucleus and, for the EL and ML conversion, also in the point-nucleus approximation. The calculated angular correlations are compared with results obtained within the Born approximation and, for the $E 0$ case, with experimental data.
\end{abstract}

\section{INTRODUCTION}

First calculations of the angular correlation in internal pair conversion (IPC) were performed by Rose for the multipolarity $L>0$ of the nuclear transition ${ }^{1}$ and by Oppenheimer for the electric monopole conversion. ${ }^{2}$ Both calculations utilize second-order perturbation theory with ordinary plane waves as lepton wave functions, i.e., Born approximation is employed. For the decay of states close to threshold in heavy nuclei one clearly should take into account that electrons and positrons are created in the Coulomb potential of the nucleus. As a consequence, the lepton wave functions will be strongly deformed by this potential which is characterized by the coupling constant $Z \alpha$. The representation of electrons and positrons by ordinary plane waves implies the neglect of any Coulomb distortion $(Z \alpha=0)$.

For a nuclear charge number $Z=40$, we have $Z \alpha \approx 0.3$. In this domain plane waves can be replaced by Sommerfeld-Maue wave functions ${ }^{3}$ which have been employed, e.g., by Bethe and Maximon ${ }^{4}$ in the calculation of angular correlation in bremsstrahlung and pair production. But let us consider a uranium nucleus which has a charge number of $Z=92$ corresponding to $Z \alpha \approx 0.7$, or even higher nuclear charges being generated for a short period of time in heavy-ion collisions that lead to a coupling constant $Z \alpha>1$. In these strong fields it is obvious that a perturbation expansion in $Z \alpha$ underlying the Sommerfeld-Maue wave functions is expected to fail.

In this paper, we completely account for the Coulomb deformation of the wave functions by utilizing exact scattering wave functions for electrons and positrons, i.e., Coulomb-distorted plane waves. Asympotically they represent plane waves, which are eigenfunctions of the momentum operator. This choice of eigenfunctions al- lows to discuss dependencies on directions and opening angles. The Coulomb-distorted wave functions are discussed in more detail in Sec. II. A theoretical description of the transition amplitude has been presented in Ref. 5 . For the sake of completeness and in order to introduce our notations a brief summary is given in Sec. II.

For nuclear transitions of angular momentum $L>0$ we neglect any penetration effects which would otherwise require assumptions about a specific nuclear model. ${ }^{5,6}$ These assumptions would drastically complicate the calculations, and furthermore, would contain uncertainties on the same scale as this "no penetration" approximation. Our numerical results are obtained assuming a finite extension of the nucleus as well as a pointlike nucleus.

A major motivation for our theoretical investigations results from attempts to explain the narrow line structures in coincident electron and positron spectra ${ }^{7}$ which have been observed by several collaborations in heavy-ion collisions below the Coulomb barrier at the UNILAC accelerator of the Gesellschaft für Schwerionenforschung in Darmstadt, Germany. The origin of these line structures is yet unknown, although many explanations were suggested. ${ }^{8}$ Nuclear effects seemed to be ruled out. ${ }^{9}$ Deltaelectron distributions as well as photon spectra have been measured simultaneously to the positron spectra. Internal conversion processes would reflect themselves in all these channels with relative intensities being determined by the associated conversion coefficients. However, it could be demonstrated experimentally that the observed narrow positron lines are not accompanied by corresponding peaks in delta-electron or photon distributions. The recent experimental setups are improved to detect electrons and positrons coincidentally with respect to their opening angle. ${ }^{10}$ In this connection it is important 
to know precisely the angular correlation of the various $e^{+} e^{-}$pair-creating processes. A major contribution besides the dynamically created electrons and positrons ${ }^{11}$ stems from internal pair conversion. ${ }^{12}$

\section{THEORY OF PAIR CONVERSION}

\section{A. Definitions}

In the following we give a brief overview of the quantities under discussion. The pair conversion coefficients are defined in the case of a nuclear transition with angular momentum $L>0$ and parity $(-)^{L}$ (electric or EL conversion) and $(-)^{L+1}$ (magnetic or ML conversion) as

$$
\beta=\frac{P_{e^{+} e^{-}}}{P_{\gamma}},
$$

i.e., as the ratio of the pair conversion probability to the total $\gamma$-emission probability. For $E 0$ conversion, in which photon emission is forbidden, we use

$$
\eta=\frac{P_{e^{+} e^{-}}}{P_{e^{-}}},
$$

i.e., the ratio of the pair conversion probability to the total probability of emitting a bound-state $K$-shell electron. The coefficients $\beta$ and $\eta$ can be expressed as integrals over the differential coefficients with respect to the kinetic positron energy $E$,

$$
\beta=\int_{0}^{\omega-2 m} d E \frac{d \beta}{d E}, \quad \frac{d \beta}{d E}=\frac{d P_{e^{+} e^{-}} / d E}{P_{\gamma}}
$$

for angular momenta $L>0$, or for $E 0$ transitions,

$$
\eta=\int_{0}^{\omega-2 m} d E \frac{d \eta}{d E}, \quad \frac{d \eta}{d E}=\frac{d P_{e^{+} e^{-}} / d E}{P_{e^{-}}},
$$

where $\omega$ is the nuclear transition energy. Denoting the angle between electron and positron with $\theta$, we write the coefficients $d \beta / d E$ and $d \eta / d E$ as integrals over the angle $\theta$ and the positron energy $E$

$$
\begin{aligned}
& \beta=\int_{0}^{\omega-2 m} d E \int_{-1}^{1} d \cos \theta \frac{d^{2} \beta}{d E d \cos \theta} \\
& \eta=\int_{0}^{\omega-2 m} d E \int_{-1}^{1} d \cos \theta \frac{d^{2} \eta}{d E d \cos \theta} .
\end{aligned}
$$

We have to evaluate the doubly differential pair conversion probability $d P_{e^{+} e^{-}}^{2} /(d E d \cos \theta)$.

\section{B. The wave functions}

The wave functions which enter into the expression for the pair conversion probability are solutions of the Dirac equation for a nuclear potential $V$ :

$$
(\boldsymbol{\alpha} \cdot \mathbf{p}+V+\beta m) \Psi=i \frac{\partial}{\partial t} \Psi .
$$

As nuclear potential $V$ we consider the Coulomb potential of a pointlike nucleus

$$
V(r)=-\frac{Z \alpha}{r}
$$

as well as the more realistic potential of an extended nucleus,

$$
V(r)=\left\{\begin{array}{l}
-Z \alpha /\left(2 R_{n}\right)\left[3-\left(r^{2} / R_{n}^{2}\right)\right] \text { for } r \leq R_{n} \\
-Z \alpha / r \text { for } r>R_{n}
\end{array}\right.
$$

treated as a homogeneously charged sphere with radius $R_{n}=1.2 \mathrm{fm} A^{1 / 3}$.

In the case of IPC, we are interested in continuum solutions of Eq. (7). We can construct various complete orthogonal sets of continuum wave functions which satisfy different boundary conditions. Solving Eq. (7) in spherical coordinates we obtain the angular momentum eigenfunctions $\chi_{W_{\kappa \mu}}$ in their well-known form

$$
\chi_{W \kappa \mu}=\left[\begin{array}{cc}
g(r) & \chi_{\kappa \mu}(\Omega) \\
i f(r) & \chi_{-\kappa \mu}(\Omega)
\end{array}\right) .
$$

$\chi_{\kappa \mu}$ are the spherical spinors which determine the angular dependence of the electron wave functions. In the pointnucleus case the radial wave functions $g$ and $f$ are given by

$$
\begin{aligned}
& u_{1}(r)=r g(r)=\frac{(|W+m|)^{1 / 2}(2 p r)^{\gamma} e^{(\pi / 2) y}|\Gamma(\gamma+i y)|}{2(\pi p)^{1 / 2} \Gamma(2 \gamma+1)}\left[e^{-i p r+i \eta}(\gamma+i y)_{1} F_{1}(\gamma+1+i y, 2 \gamma+1 ; 2 i p r)+c . c .\right] \\
& u_{2}(r)=r f(r)=\frac{i(|W-m|)^{1 / 2}(2 p r)^{\gamma} e^{(\pi / 2) y}|\Gamma(\gamma+i y)|}{2(\pi p)^{1 / 2} \Gamma(2 \gamma+1)}\left[e^{-i p r+i \eta}(\gamma+i y)_{1} F_{1}(\gamma+1+i y, 2 \gamma+1 ; 2 i p r)-c . c .\right]
\end{aligned}
$$

where $\gamma=\left[\kappa^{2}-(Z \alpha)^{2}\right]^{1 / 2}$, and $y=Z \alpha W / p$ is the Sommerfeld parameter. ${ }^{3} W$ denotes the total energy which is related for a positive-energy electron to its kinetic energy $E$ by $W=E+m$ and for a negative-energy electron to the kinetic energy of the corresponding positron by
$W=-E-m . \eta$ is part of the phase shift treated later.

Another set of solutions, which are known as Coulomb-distorted plane waves, $\psi_{\mathrm{p}, \lambda}^{( \pm)}$, can be derived by solving Eq. (7) in parabolic coordinates. ${ }^{13}$ These wave functions firstly constructed by Mott $^{14}$ are required for 
the determination of angular correlations since they represent asymptotically plane waves with definite momentum $\mathbf{p}$ and polarization $\lambda$. Calculating the conversion probability with these waves enables us to determine the angle between electron and positron direction. The
Coulomb-distorted plane waves can be decomposed into spherical Dirac waves

$$
\psi_{\mathbf{p}, \lambda}^{( \pm)}=\sum_{\kappa, \mu} \alpha_{\kappa \mu}^{( \pm)}(\Omega, \lambda) \chi_{W_{\kappa \mu}},
$$

with the expansion coefficients

$$
\begin{aligned}
a_{\kappa \mu}^{( \pm)} & =\frac{(2 l+1)^{1 / 2}}{(4 \pi)^{1 / 2}} \frac{1}{(W p)^{1 / 2}} i^{l} e^{ \pm i \delta_{\kappa}}\left(l \frac{1}{2} j \mid 0 \lambda \lambda\right) D \dot{\lambda \mu}_{\mu}(\varphi, \vartheta) \\
& =\frac{[(2 l+1)(2 j+1)]^{1 / 2}}{(4 \pi W p)^{1 / 2}} i^{l} e^{ \pm i \delta_{\kappa}}\left(\begin{array}{ccc}
l & \frac{1}{2} & j \\
0 & \lambda & -\lambda
\end{array}\right](-)^{l-\frac{1}{2}-\lambda} D_{\mu \lambda}^{j}(\varphi, \vartheta) .
\end{aligned}
$$

The coefficients depend on the Dirac quantum number $\kappa\left(|\kappa|=j+\frac{1}{2}\right)$ and spin projection $\mu$ of the electron, on the direction $\Omega$ of its momentum represented by the rotation matrix $D_{\mu \lambda}^{j}(\varphi, \vartheta)$ including the Euler angles $\vartheta$ and $\varphi,{ }^{15}$ and on its polarization $\lambda$.

The waves $\psi_{\mathrm{p} \lambda}^{(+)}$and $\psi_{\mathrm{p} \lambda}^{(-)}$differ only in the sign of the phase shift $\delta_{\kappa}$ in the argument of the exponential function which determines the asymptotic behavior. $\delta_{\kappa}$ is just the difference,

$$
\delta_{\kappa}=\delta_{\mathrm{stat}, \kappa}-\delta_{\mathrm{pl}, \kappa},
$$

of the phase shift $\delta_{\text {stat }, \kappa}$ of a stationary spherical wave $\chi_{W_{\kappa \mu}}$ without the logarithmic phase shift $\delta_{\log }=-y \ln 2 p r$ to the phase shift $\delta_{\mathrm{pl}, \kappa}$ of the corresponding term in the partial-wave decomposition of a plane wave. Thus, Eq. (15) gives the scattering phase shift of the Coulombdistorted waves. The wave functions are normalized according to the following conditions:

$$
\begin{aligned}
& \left\langle\psi_{\mathbf{p}, \lambda} \mid \psi_{\mathbf{p}, \lambda^{\prime}}\right\rangle=\frac{1}{p^{2}} \delta\left(p-p^{\prime}\right) \delta\left(\Omega-\Omega^{\prime}\right) \delta_{\lambda, \lambda^{\prime}}, \\
& \left\langle\chi_{W \kappa \mu} \mid \chi_{W^{\prime} \kappa^{\prime} \mu^{\prime}}\right\rangle=\delta_{\kappa \kappa^{\prime}} \delta_{\mu \mu^{\prime}} \delta\left(W-W^{\prime}\right) .
\end{aligned}
$$

The Coulomb-distorted waves display the asymptotic behavior

$$
\psi_{\mathbf{p} \lambda}^{( \pm)} \rightarrow N\left(\omega_{r} e^{i[\mathbf{k} \cdot \mathbf{r} \pm y \ln (k r \mp \mathbf{k} \cdot \mathbf{r})]}+f_{r}^{( \pm)} \frac{1}{r} e^{ \pm i(k r-y \ln 2 k r)}\right)
$$

as $r \rightarrow \infty . \omega_{r}$ and $f_{r}^{( \pm)}$are four-spinors. We consider the pair creation by IPC as a time-dependent process; that means we assume that electron and positron are detected at a time $t \rightarrow \infty$ after their creation at $t_{0}$ in the nuclear potential. Furthermore, we suppose the detectors to be constructed to measure the electron's or positron's momenta. Then the lepton wave functions should be represented by plane waves at $t \rightarrow \infty$, and consequently, we have to describe the electron and positron by the $\psi_{\mathrm{p} \lambda}^{(-)}$ scattering waves. ${ }^{4}$ Since we want to calculate transition amplitudes, we must transform the positron wave into a wave function which describes a negative-energy electron. We consider again a positron moving forward in time being detected at $t \rightarrow \infty$. Its time propagating phase factor reads $e^{-i \boldsymbol{W} t}(\boldsymbol{W}>m)$ and it consists of converging spherical waves corresponding to $\psi^{(-)}$which are proportional to

$$
\frac{1}{r} e^{-i(k r+W t)}
$$

(for sake of lucidity we omit the logarithmic phase shift). We may interpret this outgoing positron as an ingoing electron with the negative positron energy moving backwards in time. Equation (18) then becomes

$$
\frac{1}{r} e^{-i[k r-(-W) t]} \text {. }
$$

Since the time parameter $t$ decreases, the negative-energy electron consists of converging partial waves and is to be described by a $\psi^{(-)}$-type wave function. Thus, we must express both the positive-energy electron and the negative-energy electron by $\psi^{(-)}$wave functions using the expansion coefficients $a_{\kappa \mu}^{(-)}$. [We drop the $(-)$superscript from now on.]

Let us add some remarks on the phase shift. In the point-nucleus approximation the phase shift of the stationary waves in Eq. (15) reads as

$$
\delta_{\text {stat }, \kappa}=y \ln 2 p r-\arg \Gamma(\gamma+i y)-\frac{\pi \gamma}{2}+\eta
$$

with the Sommerfeld parameter $y$ and $\eta=\arg \sqrt{(\kappa-i y / E) /(\gamma+i y)}$. Note that some authors use a some kind different phase shift:

$$
\begin{aligned}
& \widetilde{\delta}_{\text {stat }, \kappa}=y \ln 2 p r-\arg \Gamma(\gamma+1+i y)-\frac{\pi \gamma}{2}+\eta^{\prime}, \\
& \eta^{\prime}=\arg \sqrt{(-\kappa+i y / E) /(\gamma-i y)} .
\end{aligned}
$$

Simultaneously, the normalized factors [in Eq. (11)] are multiplied with the absolute values of

$$
(\gamma \pm i y)=|\gamma+i y| e^{ \pm i \arg (\gamma+i y)} .
$$

In the case of an extended nucleus we have to compute the electron wave functions and their phase shifts numerically. Details of this procedure are given in Appendix A. 


\section{The transition amplitude}

We first calculate the $S$ matrix element which determines the pair-conversion probability in second order. The total pair-conversion probability reads as

$$
P_{e^{+} e^{-}}=\frac{2 \pi}{2 J_{i}+1} \sum_{i} \sum_{f}\left|U_{i f}\right|^{2} \delta\left(W+W^{\prime}-\omega\right) .
$$

$U_{i f}$ denotes the transition amplitude of the IPC process. Energy conservation, i.e., the fact that the nuclear transition energy $\omega$ splits into electron energy $W^{\prime}$ and positron energy $W$, is expressed by the $\delta$ function. We have to take the summation over the final states and to average over the initial states. As we are concerned with plane waves in the final channel, we sum over the momenta $p$ and $\mathbf{p}^{\prime}$ of electron and positron and their polarizations $\lambda^{\prime}$ and $\lambda$, respectively. In addition, we sum over the final states of the nucleus, i.e., the projection quantum numbers $M_{f}$. The initial states are those of the nucleus, i.e., the projection quantum numbers $M_{i} . U_{i f}$ is given in the retarded form

$$
\begin{aligned}
U_{i f}=-\alpha \int d \tau_{n} \int d \tau_{e}[ & \rho_{n}\left(\mathbf{r}_{n}\right) \rho_{e}^{\mathrm{pl}}\left(\mathbf{r}_{e}\right) \\
& \left.+\mathbf{j}_{n}\left(\mathbf{r}_{n}\right) \cdot \mathbf{j}_{e}^{\mathrm{pl}}\left(\mathbf{r}_{e}\right)\right] \frac{e^{i\left|\mathbf{r}_{n}-\mathbf{r}_{e}\right| \omega}}{\left|\mathbf{r}_{n}-\mathbf{r}_{e}\right|}
\end{aligned}
$$

The first term in the square brackets denotes the Coulomb interaction of the nuclear transition charge density $\rho_{n}$ with the electron transition charge density $\rho_{e}$. The second term describes the coupling of the nuclear and the electron transition current densities $\mathbf{j}_{n}$ and $\mathbf{j}_{e}$. The electron transition charge and current densities are expressed in terms of Coulomb-distorted plane-wave functions

$$
\rho_{e}^{\mathrm{pl}}=\psi_{\mathbf{p}^{\prime}, \lambda^{\prime}}^{\dagger} \psi_{\mathbf{p} \lambda}, \quad \mathbf{j}_{e}^{\mathrm{pl}}=\psi_{\mathbf{p}^{\prime} \lambda^{\prime}}^{\dagger} \boldsymbol{\alpha} \psi_{\mathbf{p} \lambda}
$$

The factor $G\left(\mathbf{r}_{n}, \mathbf{r}_{e}\right)=e^{l\left|\mathbf{r}_{n}-\mathbf{r}_{e}\right| \omega} /\left|\mathbf{r}_{n}-\mathbf{r}_{e}\right|$ is the photon propagator in position space. Inserting the sphericalwave decomposition we get (since the integral is sesquilinear)

$$
U_{i f}=\sum_{\kappa, \mu} \sum_{\kappa^{\prime}, \mu^{\prime}} a_{\kappa^{\prime} \mu^{\prime}}^{*} a_{\kappa \mu} U_{i f}^{\mathrm{sph}}
$$

where the transition amplitude $U_{i f}^{\text {sph }}$ is of the same form as Eq. (23), but with the Coulomb-distorted plane waves replaced by the spherical waves in Eq. (10).

Writing the electron direction $d \Omega=\sin \vartheta d \vartheta d \varphi$, and $W d W=p d p$ we get the expression for the differential pair-conversion probability:

$$
\frac{d P_{e^{+} e^{-}}}{d W}=\frac{2 \pi}{2 J_{i}+1} \sum_{M_{1}, M_{f}} \sum_{\kappa, \kappa^{\prime}, \mu, \mu^{\prime}} \sum_{k, k^{\prime}, m, m^{\prime}} \sum_{\lambda, \lambda^{\prime}} \int d \Omega \int d \Omega^{\prime} p W p^{\prime} W^{\prime} a_{\kappa^{\prime} \mu^{\prime}}^{\prime *} a_{\kappa \mu} a_{k^{\prime} m^{\prime}}^{\prime} a_{k m}^{*}\left|U_{i f}^{\mathrm{sph}}\right|^{2} .
$$

Since the electromagnetic transitions of the atomic nucleus can be classified by their angular momentum $L$ and their parity $\pi$, we split the amplitude $U_{i f}$ using the series expansion of the Green's function,

$$
G\left(\mathbf{r}_{e}, \mathbf{r}_{n}\right)=4 \pi i \omega \sum_{L=0}^{\infty} j_{L}\left(\omega r_{<}\right) h_{L}^{(1)}\left(\omega r_{>}\right) \sum_{M=-L}^{+L} Y_{L M}^{*}\left(\Omega_{n}\right) Y_{L M}\left(\Omega_{e}\right)
$$

where $r_{<}=\min \left(r_{e}, r_{n}\right)$ and $r_{>}=\max \left(r_{e}, r_{n}\right)$. Inserting the unit dyad $\mathbf{I}=\sum_{\mu} \xi_{\mu} \xi-\mu(-)^{\mu}$ into the current part of Eq. (23) one gets from Eq. (27) the dyadic Green's function

$$
\mathbf{I} G\left(\mathbf{r}_{e}, \mathbf{r}_{n}\right)=4 \pi i \omega \sum_{L, M, \tau} \mathbf{B}_{L M}^{(\tau)} \mathbf{A}_{L M}^{(\tau) *},
$$

where $\tau=e, m, l$ enumerates the electric, magnetic, and longitudinal contributions. The magnetic and electric fields ${ }^{16}$ are

$$
\begin{aligned}
& \mathbf{A}_{L M}^{(m)}\left(\omega \mathbf{r}_{n}\right)=-\sum_{\mu}(1 L L \mid-\mu m M) j_{L}\left(\omega r_{n}\right) Y_{L m}\left(\Omega_{n}\right) \xi_{-\mu} \\
& \mathbf{A}_{L M}^{(e)}\left(\omega \mathbf{r}_{n}\right)=\frac{1}{\sqrt{2 L+1}}\left(\begin{array}{l}
-\sqrt{L} \sum_{\mu}(1 L+1 L \mid-\mu m M) j_{L+1}\left(\omega r_{n}\right) Y_{L+1 m}\left(\Omega_{n}\right) \xi_{-\mu} \\
\\
\quad+\sqrt{L+1} \sum_{\mu}(1 L-1 L \mid-\mu m M) j_{L-1}\left(\omega r_{n}\right) Y_{L-1 m}\left(\Omega_{n}\right) \xi_{-\mu}
\end{array}\right) .
\end{aligned}
$$

$\xi_{\mu}$ are the spherical basis vectors and $j_{L}\left(\omega r_{n}\right)$ denote the spherical Bessel functions. We also need the longitudinal field

$$
\begin{aligned}
& \mathbf{A}_{L M}^{(l)}\left(\omega \mathbf{r}_{n}\right)=\frac{1}{\sqrt{2 L+1}}\left(\sqrt{L+1} \sum_{\mu}(1 L+1 L \mid-\mu m M) j_{L+1}\left(\omega r_{n}\right) Y_{L+1 m}(\Omega) \xi_{-\mu}\right. \\
& \left.+\sqrt{L} \sum_{\mu}(1 L-1 L \mid-\mu m M) j_{L-1}\left(\omega r_{n}\right) Y_{L-1 m}(\Omega) \xi_{-\mu}\right] \text {. }
\end{aligned}
$$


The $\mathbf{B}_{L M}$ fields follow by replacing the spherical Bessel functions $j_{L}\left(\omega r_{n}\right)$ by the spherical Hankel functions $h_{L}^{(1)}\left(\omega r_{n}\right)$.

The transition amplitude $U_{i f}^{\text {sph }}$ now can be split into magnetic matrix elements which describe transitions of parity $\pi=(-)^{L+1}$, electric matrix elements which describe transitions of parity $\pi=(-)^{L}$, and an $E 0$ matrix element. The latter has no corresponding $\gamma$-emission amplitude as mentioned already in the beginning of this section:

$$
U_{i f}^{\mathrm{sph}}=\sum_{L>0, M}\left\{U_{i f}^{(e)}(L, M)+U_{i f}^{(m)}(L, M)\right\}+U_{i f}^{(L=0)}
$$

We consider first one of the magnetic parts. It can be written as

$$
\begin{aligned}
U_{i f}^{(m)}=4 \pi i \alpha \omega\{ & \left\{\int_{0}^{\infty} d \tau_{n} \int_{0}^{\infty} d \tau_{e}\left[\mathbf{j}_{n}\left(\mathbf{r}_{n}\right) \cdot \mathbf{A}_{L M}^{(m) *}\left(\omega \mathbf{r}_{n}\right)\right]\left[\mathbf{j}_{e}\left(\mathbf{r}_{e}\right) \cdot \mathbf{B}_{L M}^{(m)}\left(\omega \mathbf{r}_{e}\right)\right]\right. \\
& -\int_{0}^{\infty} d \tau_{n} \int_{0}^{r_{n}} d \tau_{e}\left[\mathbf{j}_{n}\left(\mathbf{r}_{n}\right) \cdot \mathbf{A}_{L M}^{(m) *}\left(\omega \mathbf{r}_{n}\right)\right]\left[\mathbf{j}_{e}\left(\mathbf{r}_{e}\right) \cdot \mathbf{B}_{L M}^{(m)}\left(\omega \mathbf{r}_{e}\right)\right] \\
& \left.+\int_{0}^{\infty} d \tau_{n} \int_{0}^{r_{n}} d \tau_{e}\left[\mathbf{j}_{n}\left(\mathbf{r}_{n}\right) \cdot \mathbf{B}_{L M}^{(m) *}\left(\omega \mathbf{r}_{n}\right)\right]\left[\mathbf{j}_{e}\left(\mathbf{r}_{e}\right) \cdot \mathbf{A}_{L M}^{(m)}\left(\omega \mathbf{r}_{e}\right)\right]\right\}
\end{aligned}
$$

Note that the asterisk used in relation with the fields $\mathbf{B}_{L M}^{(m)}$ means just the complex conjugation of the spherical harmonics rather than the complex conjugation of the (complex) Hankel functions. In the first term, denoted as a static part, the nuclear and the electron matrix element factorize. The nuclear matrix element exactly coincides with the transition amplitude of $\gamma$ emission (Appendix B):

$$
V_{\gamma}^{(m)}(L, M)=\int_{0}^{\infty} d \tau_{n} \mathbf{j}_{n}\left(\mathbf{r}_{n}\right) \cdot \mathbf{A}_{L M}^{(m) *}\left(\omega \mathbf{r}_{n}\right)
$$

The second and third terms in Eq. (33) describe the penetration effect. This expression is referred to as dynamic matrix element. Its calculation requires assumptions about a nuclear model. In Refs. 5 and 6 some methods are presented. The simplest model is the point- nucleus approximation. The electron integrals of the last two terms then vanish and the dynamic matrix element becomes zero. In this article we adopt the "no penetration" approximation of Rose, ${ }^{5}$ i.e., we evaluate the static part assuming an extended nucleus and simply neglect the dynamic matrix elements. This approximation will simplify the further calculation considerably. After squaring the matrix element $U_{i f}^{\text {sph }}$ and dividing by the $\gamma$ emission probability, the pair-conversion coefficient becomes independent of the unknown nuclear matrix element. If we were to consider strongly deformed nuclei, we would have to take the penetration effects into account. We point out that an exact treatment of the dynamic matrix element stands out yet.

Now we turn to the electric matrix element. It consists of scalar, electric $(e)$, and longitudinal $(l)$ parts and can be written, after some transformations, as

$$
\begin{aligned}
& U_{i f}^{(e)}=\frac{4 \pi i \alpha \omega}{\sqrt{L(L+1)}}\left[\int_{0}^{\infty} d \tau_{n} \int_{0}^{\infty} d \tau_{e}\left[\mathbf{j}_{n}\left(\mathbf{r}_{n}\right) \cdot \mathbf{A}_{L M}^{(e) *}\left(\omega \mathbf{r}_{n}\right)\right]\left[\omega \mathbf{j}_{e} \cdot \hat{\mathbf{r}}_{e}+i \rho_{e} \frac{\partial}{\partial r_{e}}\right) r_{e} h_{L}^{(1)}\left(\omega r_{e}\right) Y_{L M}\left(\Omega_{e}\right)\right. \\
&-\int_{0}^{\infty} d \tau_{n} \int_{0}^{r_{n}} d \tau_{e}\left[\mathbf{j}_{n}\left(\mathbf{r}_{n}\right) \cdot \mathbf{A}_{L M}^{(e) *}\left(\omega \mathbf{r}_{n}\right)\right]\left[\omega \mathbf{j}_{e} \cdot \hat{\mathbf{r}}_{e}+i \rho_{e} \frac{\partial}{\partial r_{e}}\right] r_{e} h_{L}^{(1)}\left(\omega r_{e}\right) Y_{L M}\left(\Omega_{e}\right) \\
&\left.+\int_{0}^{\infty} d \tau_{n} \int_{0}^{r_{n}} d \tau_{e}\left[\mathbf{j}_{n}\left(\mathbf{r}_{n}\right) \cdot \mathbf{B}_{L M}^{(e) *}\left(\omega \mathbf{r}_{n}\right)\right]\left[\omega \mathbf{j}_{e} \cdot \hat{\mathbf{r}}_{e}+i \rho_{e} \frac{\partial}{\partial r_{e}}\right] r_{e} j_{L}\left(\omega r_{e}\right) Y_{L M}\left(\Omega_{e}\right)\right] .
\end{aligned}
$$

In the first term, again denoted as a static part, nuclear and electric matrix elements factorize. The last two terms describe the penetration effects. We restrict ourselves to the static part. The nuclear factor appears identically in the electron $\gamma$-emission amplitude

$$
V_{\gamma}^{(e)}(L, M)=\int_{0}^{\infty} d \tau_{n} \mathbf{j}_{n}\left(\mathbf{r}_{n}\right) \cdot \mathbf{A}_{L M}^{(e) *}\left(\omega \mathbf{r}_{n}\right)
$$

Finally, the $E O$ matrix element which includes only scalar parts can be cast in simple form ${ }^{17}$

$$
U_{i f}^{(L=0)}=-\alpha \int_{0}^{\infty} d \tau_{n} \int_{0}^{r_{n}} d \tau_{e} \rho_{n}\left(\mathbf{r}_{n}\right) \rho_{e}\left(\mathbf{r}_{e}\right)\left(\frac{1}{r_{n}}-\frac{1}{r_{e}}\right)
$$

This expression shows that the $E 0$ conversion takes place by penetration effects only: That part of the electron density which is contained inside the nucleus contributes to the integral. Since for EL and ML conversion with $L>0$, we deal only with the static matrix elements we are able to write the electric and magnetic amplitudes as a 
product of the $\gamma$-emission part and electric matrix element,

$$
\begin{aligned}
& U_{i f}^{(\tau)}(L, M)= 4 \pi i \alpha \omega V_{\gamma}^{(\tau)}(L, M) M^{\tau}(L, M) \\
&= 4 \pi i \alpha \omega(-1)^{J_{f}-M_{f}}\left(\begin{array}{ccc}
J_{f} & L & J_{i} \\
-M_{f} & M & M_{i}
\end{array}\right) \\
& \times(-1)^{J^{\prime}-\mu^{\prime}}\left(\begin{array}{ccc}
j & j^{\prime} & L \\
\mu & \mu^{\prime} & M
\end{array}\right) V_{\gamma}^{(\tau)}(L) M^{\tau}(L)_{i} \\
& \tau=e, m
\end{aligned}
$$

where we made use of the Wigner-Eckart theorem. $V_{\gamma}^{\tau}(L)$ and $\boldsymbol{M}^{\tau}(L)$ are the reduced matrix elements. The electron matrix element $M^{\tau}(L)$ can be decomposed into an angular and a radial integral

$$
M^{\tau}(L)=-i \frac{\left\langle\kappa^{\prime} \| Y_{L}|| \kappa\right\rangle}{[L(L+1)]^{1 / 2}} R_{\kappa \kappa^{\prime}}^{\tau}(L),
$$

with

$$
\begin{aligned}
\left\langle\kappa^{\prime}\left\|Y_{L}\right\| \kappa\right\rangle= & (-)^{j^{\prime}+1 / 2} \sqrt{(2 j+1)\left(2 j^{\prime}+1\right)(2 L+1) / 4 \pi} \\
& \times\left(\begin{array}{ccc}
j & j^{\prime} & L \\
-\frac{1}{2} & \frac{1}{2} & 0
\end{array}\right)
\end{aligned}
$$

and the parity selection rules

$l+l^{\prime}+L+\lambda(\tau)=0 \bmod 2 \cdots\left\{\begin{array}{l}\lambda=0 \text { for } \tau=e \\ \lambda=1 \text { for } \tau=m\end{array}\right.$.

The remaining radial integrals are of the form

$$
R_{\kappa \kappa^{\prime}}^{e}(L)=L\left(R_{1}+R_{2}+R_{3}-R_{4}\right)+\left(\kappa-\kappa^{\prime}\right)\left(R_{3}+R_{4}\right)
$$

in the case of electric IPC, and

$$
R_{\kappa \kappa^{\prime}}^{m}(L)=\left(\kappa+\kappa^{\prime}\right)\left(R_{5}+R_{6}\right)
$$

for magnetic pair conversion. The radial integrals are presented as follows:

$$
\begin{aligned}
& R_{1}=\int_{0}^{\infty} d r r^{2} g_{\kappa} g_{\kappa^{\prime}}^{\prime} h_{L}^{(1)}(\omega r), \\
& R_{2}=\int_{0}^{\infty} d r r^{2} f_{\kappa} f_{\kappa^{\prime}}^{\prime} h_{L}^{(1)}(\omega r), \\
& R_{3}=\int_{0}^{\infty} d r r^{2} f_{\kappa} g_{\kappa^{\prime}}^{\prime} h_{L-1}^{(1)}(\omega r), \\
& R_{4}=\int_{0}^{\infty} d r r^{2} g_{\kappa} f_{\kappa^{\prime}}^{\prime} h_{L}^{(1)}-1(\omega r), \\
& R_{5}=\int_{0}^{\infty} d r r^{2} f_{\kappa} g_{\kappa^{\prime}}^{\prime} h_{L}^{(1)}(\omega r), \\
& R_{6}=\int_{0}^{\infty} d r r^{2} g_{\kappa} f_{\kappa^{\prime}}^{\prime} h_{L}^{(1)}(\omega r) .
\end{aligned}
$$

These have to be evaluated numerically. An extensive description of the derivation is given in Ref. 17 .

\section{Calculation of the conversion probabilities}

We insert in Eq. (26) the explicit forms of the electron wave functions, Eqs. (12) and (14), and get-see Appen$\operatorname{dix} \mathbf{D}$ for the calculational steps - the doubly differential pair-conversion probability as the integrand of

$$
\frac{d P_{e^{+} e^{-}}}{d E}=\int d E d \cos \theta \frac{d^{2} P_{e^{+} e^{-}}}{d E d \cos \theta},
$$

where we performed the trivial transformation from the total energy $W$ to the kinetic energy $E$. Dividing by the total $\gamma$-emission probability (Appendix B) this yields the expression for the angular correlation

$$
\frac{d^{2} \beta}{d E d \cos \theta} \sum_{I=0}^{\infty} a_{I} P_{I}(\cos \theta)
$$

where $P_{I}(\cos \theta)$ are the Legendre polynomials and the coefficients $a_{I}$ read as

$$
\begin{aligned}
& a_{I}=8 \pi \alpha \omega \frac{(-)^{L+1}}{L(L+1)} \sum_{\kappa, \kappa^{\prime}, k, k^{\prime}}\left|\kappa \kappa^{\prime} k k^{\prime}\right| e^{i\left(\delta_{\kappa^{\prime}}^{\prime}-\delta_{\kappa}-\delta_{k^{\prime}}^{\prime}+\delta_{k}\right)}{ }_{i}^{l^{\prime}-l-r^{\prime}+r} R_{\kappa \kappa^{\prime}}(L) R_{k k^{\prime}}^{*}(L) \\
& \times\left[\begin{array}{ccc}
j & j^{\prime} & L \\
\frac{1}{2} & -\frac{1}{2} & 0
\end{array}\right]\left(\begin{array}{ccc}
s & s^{\prime} & L \\
\frac{1}{2} & -\frac{1}{2} & 0
\end{array}\right)(2 I+1)\left(\begin{array}{ccc}
j^{\prime} & s^{\prime} & I \\
\frac{1}{2} & -\frac{1}{2} & 0
\end{array}\right)\left[\begin{array}{ccc}
j & s & I \\
\frac{1}{2} & -\frac{1}{2} & 0
\end{array}\right)\left\{\begin{array}{ccc}
j & I & s \\
s^{\prime} & L & j^{\prime}
\end{array}\right\} .
\end{aligned}
$$

In addition, we have the selection rules

$$
r^{\prime}+l^{\prime}+I=0 \bmod 2, r+l+I=0 \bmod 2
$$

and the triangle inequalities

$$
\delta\left(r^{\prime} s^{\prime} \frac{1}{2}\right), \quad \delta\left(j^{\prime} l^{\prime} \frac{1}{2}\right), \quad \delta\left(r s \frac{1}{2}\right), \quad \delta\left(j l \frac{1}{2}\right) .
$$

After integrating over the remaining angle $\theta$ we identify $a_{0}=\frac{1}{2} d \beta / d E$ and, finally, get

$$
\frac{d^{2} \beta}{d E d \cos \theta}=\frac{1}{2} \frac{d \beta}{d E}\left[1+\sum_{I>0} \frac{a_{I}}{a_{0}} P_{I}(\cos \theta)\right] .
$$

For calculating the angular correlation for the $E 0$ conversion we start with the transition amplitude for $E 0$ conversion

$$
U_{i f}=\sum_{\kappa, \mu} \sum_{\kappa^{\prime}, \mu^{\prime}} a_{\kappa^{\prime} \mu^{\prime}}^{*} a_{\kappa \mu} U_{i f}^{(L=0)}
$$


and consider the matrix element $U_{i f}^{(L=0)}$ [Eq. (37)]. Because of the orthonormality of the spinors $\int d \Omega \chi_{\kappa \mu} \chi_{\kappa^{\prime} \mu^{\prime}}=\delta_{\kappa \kappa^{\prime}} \delta_{\mu \mu^{\prime}}$ we are able to perform the angular integration involving the angular parts of the electron wave functions and we are left with

$$
U_{i f}^{(L=0)}=-\alpha \int_{0}^{\infty} d \tau_{n} \rho_{n}\left(\mathbf{r}_{n}\right) \int_{0}^{r_{n}} d r_{e} r_{e}^{2}\left(f_{\kappa} f_{\kappa}^{\prime}+g_{\kappa} g_{\kappa}^{\prime}\right)\left(\frac{1}{r_{n}}-\frac{1}{r_{e}}\right) .
$$

Repeating the steps that were applied to Eq. (23) we get

$$
\frac{d P_{e^{+} e^{-}}}{d E}=\frac{2 \pi}{2 J_{i}+1} \sum_{M_{i}, M_{f}} \sum_{\kappa, \kappa^{\prime}} \sum_{\mu, \mu^{\prime}} \sum_{\lambda, \lambda^{\prime}} \int d \Omega \int d \Omega^{\prime} a_{\kappa^{\prime} \mu^{\prime}}^{\prime *} a_{\kappa^{\prime} \mu^{\prime}} a_{\kappa \mu}^{\prime} a_{\kappa \mu}^{*}\left|U_{i f}^{(L=0)}\right| .
$$

Since $E 0$ conversion is a pure penetration effect, Eq. (52) does not split up into a nuclear and an electric matrix element. In Ref. 19 it is shown that by a skillful approximation a factorization becomes possible.

Equation (52) contains an integration over those parts of the radial electron wave functions which are inside the nucleus. In this region the wave functions can be represented by their series expansion (Appendix A). Thus, we get

$$
f_{\kappa} f_{\kappa}^{\prime}+g_{\kappa} g_{\kappa}^{\prime}=C_{\kappa} r^{2 j-1}\left(1+\sum_{i=1}^{\infty} c_{i} r^{2 k}\right),
$$

with the series coefficients $c_{i}$ and the normalization factors

$$
C_{\kappa}= \begin{cases}\lim _{r \rightarrow 0} \frac{f_{\kappa} f_{\kappa}^{\prime}}{r^{2 j-1}} & \text { for } \kappa>0 \\ \lim _{r \rightarrow 0} \frac{g_{\kappa} g_{\kappa}^{\prime}}{r^{2 j-1}} & \text { for } \kappa<0 .\end{cases}
$$

Inserting Eqs. (52) and (54) into Eq. (53) leads to

$$
\frac{d P_{e^{+} e^{-}}}{d E}=\frac{\pi \alpha^{2}}{18}|M|^{2} \sum_{\kappa, \kappa^{\prime}} \sum_{\mu, \mu^{\prime}} \sum_{\lambda, \lambda^{\prime}} \int d \Omega \int d \Omega^{\prime} a_{\kappa^{\prime} \mu^{\prime}}^{*} a_{\kappa^{\prime} \mu^{\prime}} a_{\kappa \mu}^{\prime} a_{\kappa \mu}^{*} C_{\kappa} C_{\kappa^{\prime}} .
$$

Here we set $M=\int_{0}^{\infty} d \tau_{n} \rho_{n}\left(\mathbf{r}_{n}\right) r_{n}^{2}=\left\langle r_{n}^{2}\right\rangle$ assuming that higher moments of the nuclear charge distribution can be neglected $\left(\left\langle r_{n}^{k}\right\rangle=0\right.$ for $\left.\left.k\right\rangle 2\right)$. This restricts us to angular momenta $j=\frac{1}{2}$, i.e., $\kappa= \pm 1$. Inserting the explicit form of the expansion coefficients [Eq. (13)] we obtain

$$
\begin{array}{r}
\frac{d P_{e^{+} e^{-}}}{d E}=\frac{\pi \alpha^{2}|M|^{2}}{18(4 \pi)^{2}} \int d \Omega \int d \Omega^{\prime} \sum_{\kappa, \kappa^{\prime}} \sum_{\mu, \mu^{\prime}} \sum_{\lambda, \lambda^{\prime}}(2 l+1)\left(2 l^{\prime}+1\right) e^{i \Delta_{\kappa \kappa^{\prime}}} C_{\kappa} C_{\kappa^{\prime}}\left(l \frac{1}{2} j \mid 0 \lambda^{\prime} \lambda^{\prime}\right)\left(l \frac{1}{2} j \mid 0 \lambda \lambda\right)\left(l^{\prime} \frac{1}{2} j^{\prime} \mid 0 \lambda^{\prime} \lambda^{\prime}\right) \\
\times\left(l^{\prime} \frac{1}{2} j^{\prime} \mid 0 \lambda \lambda\right) D_{\mu \lambda^{\prime}}^{j^{\prime}}\left(\varphi^{\prime}, \vartheta^{\prime}\right) D_{\mu \lambda}^{J}(\varphi, \vartheta) D_{\mu^{\prime} \lambda^{\prime}}^{j^{\prime}}\left(\varphi^{\prime}, \vartheta^{\prime}\right) D_{\mu^{\prime} \lambda}^{j^{\prime *}}(\varphi, \vartheta)
\end{array}
$$

with $\Delta_{\kappa \kappa^{\prime}}:=\delta_{\kappa}^{\prime}-\delta_{\kappa}-\delta_{\kappa^{\prime}}^{\prime}+\delta_{\kappa^{\prime}}$. A straightforward calculation yields

$$
\frac{d P_{e^{+} e^{-}}}{d E}=\frac{\pi \alpha^{2}|M|^{2}}{9(4 \pi)^{2}} \int d \Omega \int d \Omega^{\prime}\left(C_{-1}^{2}+C_{+1}^{2}-4 C_{-1} C_{+1} \cos \Delta_{+1-1} \cos \theta\right) \text {. }
$$

Again, no direction in space is singled out. This allows us to perform the integration over, for instance, the direction $\Omega$ and the azimuthal angle $\varphi^{\prime}$. The remaining integrand is the doubly differential pair-conversion probability

$$
\begin{aligned}
\frac{d^{2} P_{e^{+} e^{-}}}{d E \cos \theta}= & \frac{1}{2} \frac{\pi \alpha^{2}|M|^{2}}{9}\left(C_{-1}^{2}+C_{+1}^{2}\right) \\
& \times\left(1-2 \frac{C_{-1} C_{+1}}{C_{-1}^{2}+C_{+1}^{2}} \cos \Delta_{+1-1} \cos \theta\right) .
\end{aligned}
$$

Dividing by the total probability of the emission of a $K$ - shell electron, ${ }^{19}$

$$
P_{e^{-}}=\frac{\pi \alpha^{2}}{9}|M|^{2}\left|C_{K}\right|^{2},
$$

we find that the nuclear matrix elements in Eqs. (59) and (60) cancel exactly. With differential pair-conversion probability for $E 0$ conversion $d \eta / d E=\left(C_{+1}^{2}+C_{-1}^{2}\right) /$ $\left|C_{K}\right|^{2}$ we finally get

$$
\begin{aligned}
& \frac{d^{2} \eta}{d E \cos \theta}=\frac{1}{2} \frac{d \eta}{d E}(1+\epsilon \cos \theta) \\
& \epsilon=-2 \frac{C_{-1} C_{+1}}{C_{-1}^{2}+C_{+1}^{2}} \cos \Delta_{+1-1} .
\end{aligned}
$$


(If the $K$ shell is imbedded into the negative-energy continuum as expected for nuclear charge numbers $Z \geq 173$ one may replace $C_{K}$ by $C_{L}$, thus normalizing $\eta$ to the probability of emitting an $L$-shell electron.) The anisotropy factor is bounded: $-1 \leq \epsilon \leq 1$. Clearly, for $\epsilon=0$ the angular correlation is isotropic.

\section{DISCUSSION OF THE RESULTS}

In this section we restrict ourselves to the discussion of the anisotropy parts of Eqs. (50) and (61) since the angleintegrated differential pair-conversion coefficients have been treated extensively in Ref. 17. The coefficients are also tabulated in Ref. 20. First we present the results for $E 0$ conversion. Figure 1 shows the dependence of the anisotropy factor $\epsilon$ on the positron energy for the $0^{+} \rightarrow 0^{+}$ transition in ${ }_{40}^{90} \mathrm{Zr}$ with a transition energy $\omega=1760.7$ $\mathrm{keV}$. In addition, the anisotropy factor derived in Born approximation is plotted in Fig. 1 (dashed line). In both cases $\epsilon$ takes its maximum if the nuclear transition is shared equally among electron and positron. The angular correlation [Eq. (61)] exhibits its maximum at an opening angle $\theta=0^{\circ}$. For strongly asymmetric splitting of the nuclear transition energy the angular distribution becomes isotropic. We emphasize the close agreement between the results of our calculations with those obtained within the Born approximation as it was expected for low nuclear charges and intermediate transition energies. Figure 2 shows the anisotropy coefficient computed for a uranium-like nucleus and a low nuclear transition energy $\omega=1300 \mathrm{keV}$. One recognizes that the exact anisotropy coefficient exceeds the Born approximation result for almost all positron energies.

In Fig. 3 we plot $\epsilon$ versus the positron energy for $E 0$ conversion in various hypothetical superheavy nuclei at a near-critical nuclear charge number $Z=170$ and several supercritical charges. The transition energy was assumed to be $\omega=2000 \mathrm{keV}$. Obviously the anisotropy factor is very asymmetric in the critical case. In the supercritical case $\epsilon$ contains one or two zeros at which the differential conversion coefficient $d \eta / d E$ is strongly peaked (Fig. 4). ${ }^{21}$ A brief look at Eq. (61) explains this phenomenon:

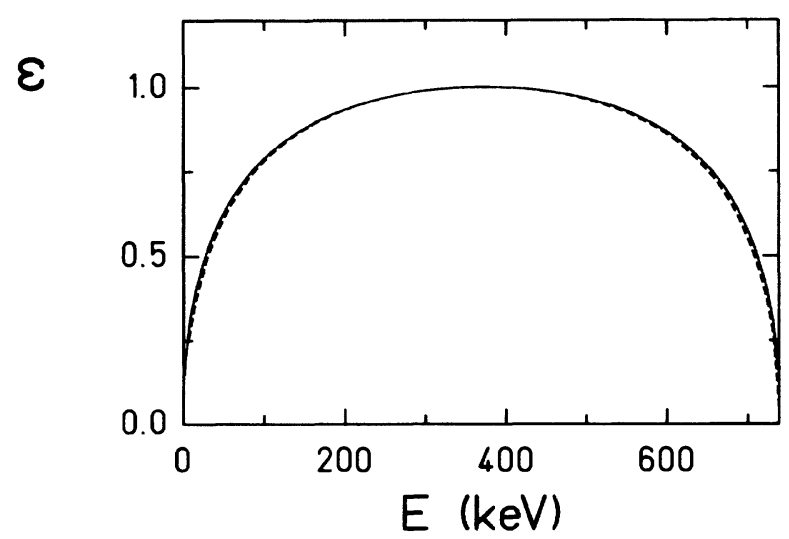

FIG. 1. Anisotropy factor $\epsilon$ in dependence on the kinetic positron energy $E$ for ${ }_{40}^{90} \mathrm{Zr}$. The $\mathrm{O}^{+} \rightarrow \mathrm{O}^{+}$transition has an energy $\omega=1760.7 \mathrm{keV}$. The dashed curve represents the result obtained within the Born approximation.

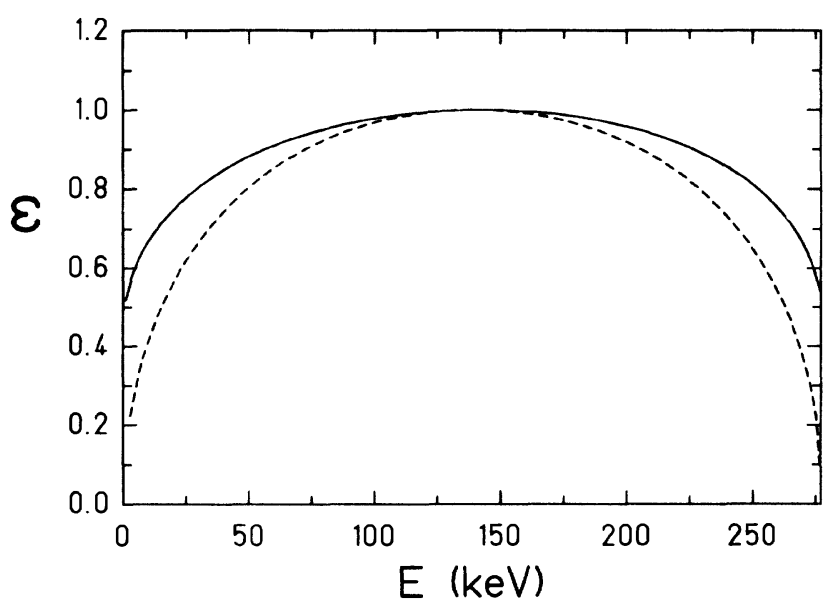

FIG. 2. Same as in Fig. 1 but assuming $E 0$ conversion of a uraniumlike nucleus with a transition energy of $\omega=1300 \mathrm{keV}$. Again the dashed curve belongs to the Born approximation result.

The large values of $d \eta / d E$ arise from peaks of either $C_{+1}^{2}$ or $C_{-1}^{2}$, compared to which the interference term $C_{+1} C_{-1}$ entering the anisotropy factor $\epsilon$ is suppressed. These quantities are related to the electron density at the origin. The sudden increase of the electron density is a resonance behavior, which occurs when a bound electron state dives into the negative-energy continuum. ${ }^{22}$ At a resonance the phase shift of the wave function of the negative-energy electron changes rapidly by $\pi$. This causes a change of the sign of $\cos \Delta_{+1-1}$ and, therefore, the change of the sign of the anisotropy factor $\epsilon$. The zeros of $\epsilon$ and peaks of $d \eta / d E$ are located at the resonance energies. For $Z=188$ both $j=\frac{1}{2}$ states $\left(1 s_{1 / 2}\right.$ and $2 p_{1 / 2}$ ) are imbedded into the negative-energy continuum. Note that the preceding discussion is quite hypothetical since it assumes that the supercritical nucleus is stable for a time interval larger than the inverse width of the resonance $\left(\sim 10^{-19} \mathrm{~s}\right)$.

Figure 5 displays the angular correlation integrated over all positron energies versus the opening angle $\theta$ for

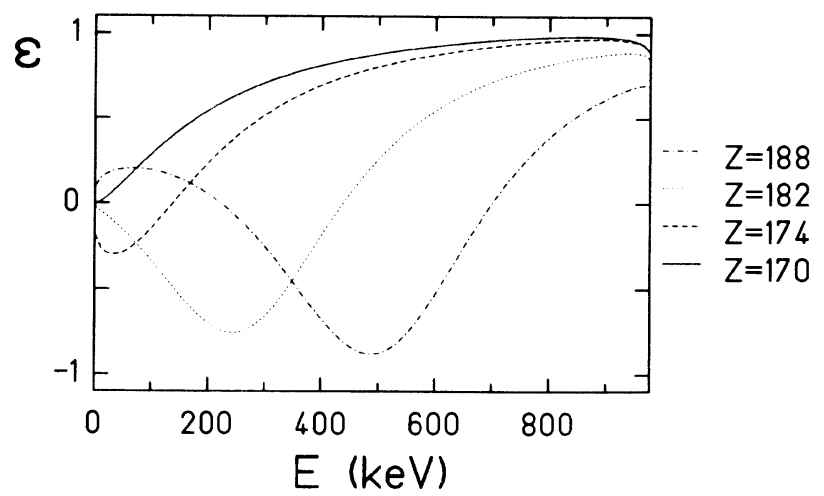

FIG. 3. Anisotropy factor $\epsilon$ plotted versus the kinetic positron energy $E$ for the $E 0$ conversion of a hypothetic nucleus with a nuclear charge number $Z=170$ near the critical one $(Z \approx 173)$ and for supercritical nuclear charge numbers. The zeros occur at the resonance energies in the supercritical cases. $(\omega=2000 \mathrm{keV}$. $)$ 


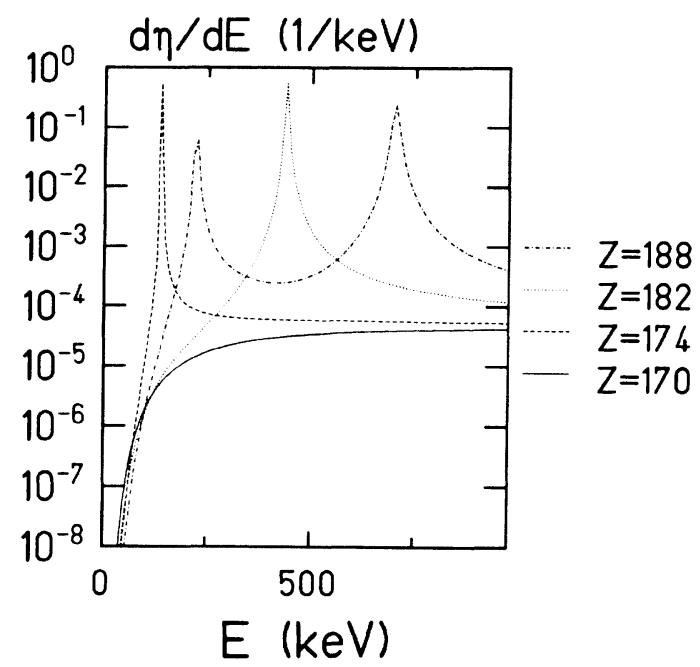

FIG. 4. Differential pair-conversion coefficient $d \eta / d E$ plotted versus the kinetic positron energy $E$ for the same nuclear charge numbers as in Fig. 3. At the resonance energies sharp maxima of $d \eta / d E$ can be observed.

the $0^{+} \rightarrow 0^{+}$transition in ${ }^{16} \mathrm{O}(\omega=6 \mathrm{MeV})$. The result of our calculation, plotted as a solid line in Fig. 5, corresponds to an anisotropy factor $\epsilon=1.0$ and is in good agreement with the experimental result. ${ }^{23}$ At large angles the data show a deviation from our result, which agrees fully with the Born approximation (not plotted) in this case, the anisotropy factor being $15 \%$ smaller than expected $(\epsilon=0.85)$. The origin of this discrepancy is presently not understood.

Having discussed the $E 0$ case the results obtained for the electric and magnetic pair conversion are immediately understandable. We are able to compute the angular correlation for point nuclei and extended nuclei. The difference manifests itself in the evaluation of the radial integrals, Eq. (44). While the integration in the case of an extended nucleus is a purely numeric one (since the wave functions are only given numerically), ${ }^{17}$ the integrals Eq. (44), in the point nucleus case can be treated analytically to give a recursion formula which then is evaluated numerically. ${ }^{18}$ As a check of our numerical methods we have calculated the angular distribution for conversion of various multipolarities in the limiting case $Z=0$. We

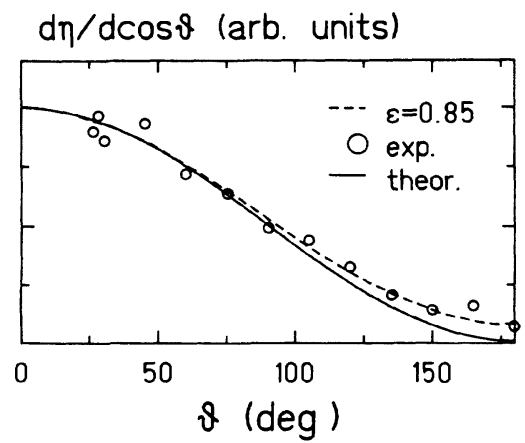

FIG. 5. Calculation of the angular correlation integrated over all positron energies compared to experimental results for the $0^{+} \rightarrow 0^{+}$transition in ${ }^{16} \mathrm{O}$ with $\omega=6 \mathrm{MeV}$. The dashed curves is fitted with an anisotropy coefficient $\epsilon=0.85$ (Ref. 23).

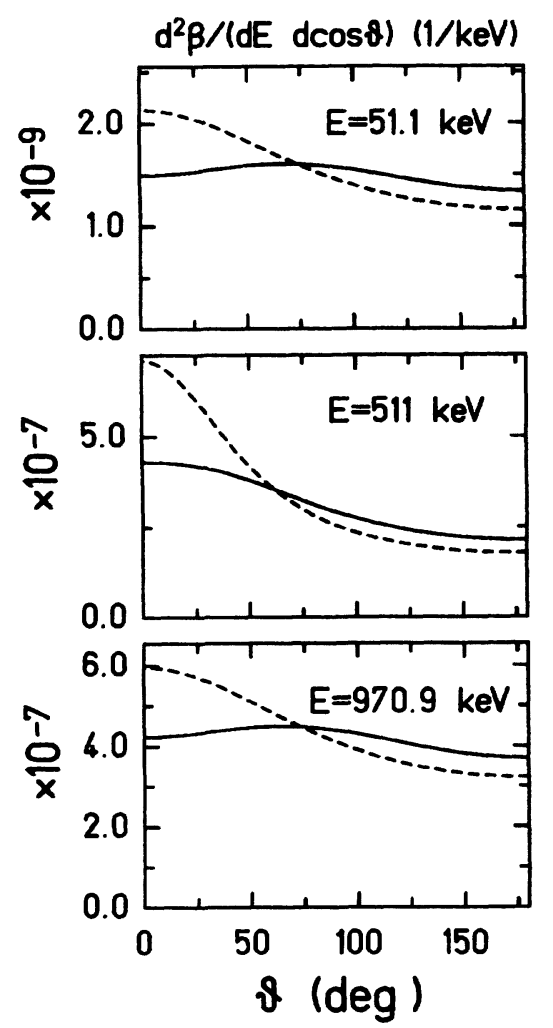

FIG. 6. Angular correlation computed for an assumed $E 1$ conversion of uranium with a transition energy $\omega=2046 \mathrm{keV}$ and three kinetic positron energies. The dashed curve shows the results obtained within the Born approximation.

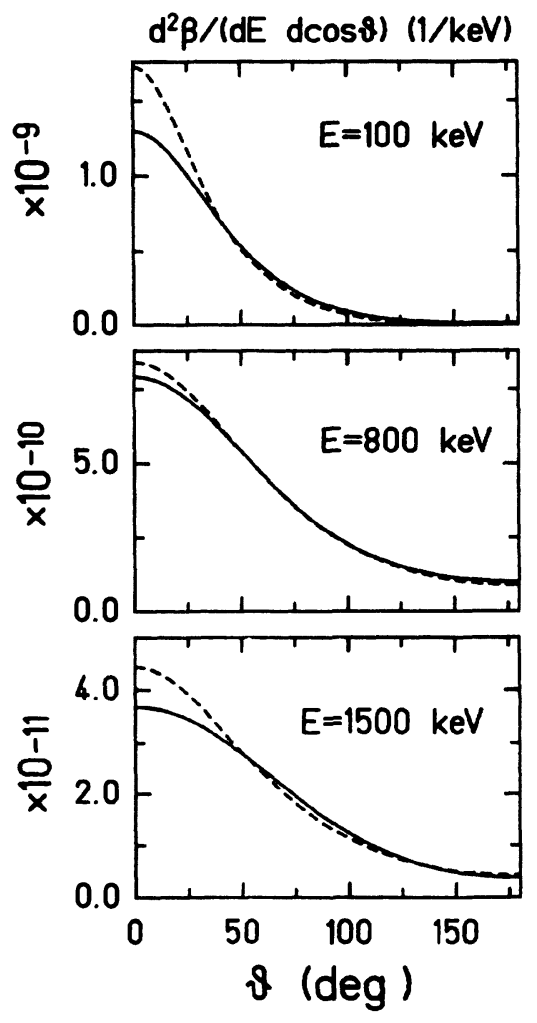

FIG. 7. Same as in Fig. 6 for the $E 3$ transition of ${ }_{82}^{208} \mathrm{~Pb}$. The transition energy amounts to $\omega=2615 \mathrm{keV}$. 


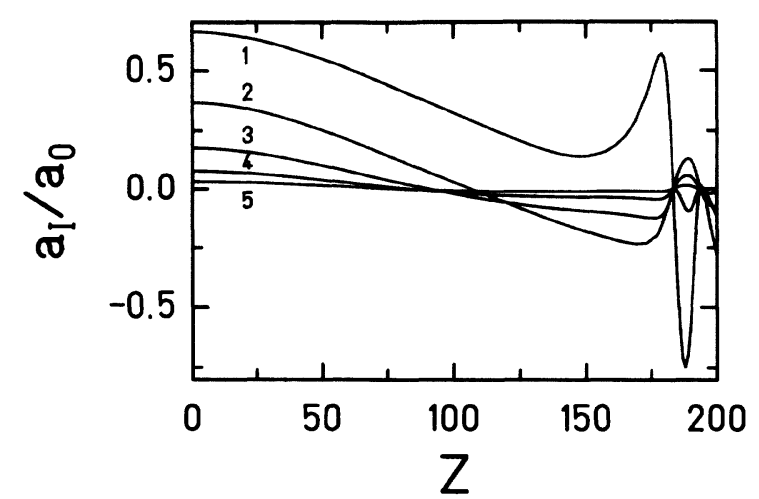

FIG. 8. Anisotropy coefficients $a_{I} / a_{0}$ plotted in dependence on the nuclear charge number for $E 1$ transition energy $\omega=2046$ $\mathrm{keV}$ and a kinetic positron energy $E=511 \mathrm{keV}$. The calculations are performed assuming a finite extension of the nucleus.

found deviations less than $0.1 \%$ compared to the results derived by Rose using the Born approximation (Appen$\operatorname{dix}$ C).

We are able to compute the angular correlation for multipolarities $L \leq 5$ and for nuclear charge numbers up to $Z=200$. In the following we pick out two examples. The first is the angular correlation assuming an $E 1$ transition of a uraniumlike nucleus for three positron energies and a transition energy $\omega=2046 \mathrm{keV}$ compared to the results in the Born approximation (Fig. 6). The Born approximation is more strongly peaked around an opening angle $\theta=0^{\circ}$ in all cases. The angular distribution obtained from our calculation is more isotropic.

Next we consider in Fig. 7 a more realistic case, namely the known $E 3$ transition in ${ }_{82}^{208} \mathrm{~Pb}$ with a transition energy of $\omega=2615 \mathrm{keV}$. Again we plotted the angular distribution for three positron energies. In Fig. 8 we plotted the dependence of the normalized anisotropy coefficients $a_{I} / a_{0}, I=1, \ldots, 5$ on the nuclear charge number $Z$ for a transition energy $\omega=2046 \mathrm{keV}$ and a kinetic energy of the positron $E=511 \mathrm{keV}$. The slope of the curves shows the deviation from the $Z$-independent Born approximation when the nuclear charge number is increased. In the supercritical region one recognizes the zeros of the curves which point to resonant states imbedded into the negative energy continuum. Again this is explained by an increase of the differential conversion coefficient $d \beta / d E \equiv a_{0}$ which suppresses the anisotropy coefficients $a_{i} / a_{0}$. In contrast to $E 0$ conversion the anisotropy coefficients for EL and ML conversion with $L>0$ do not necessarily change their signs at these points because of the great number of contributing terms only some of which contain the resonant-state wave function.

\section{CONCLUSION AND OUTLOOK}

We have calculated the angular correlation of electrons and positrons in second-order perturbation theory using the exact scattering wave functions for electrons and positrons. With the use of these wave functions the influence of the Coulomb potential of the nucleus is taken into account. Thus, we get expressions for the anisotropy fac- tors which describe the angular correlation and for the differential conversion coefficients which describe the spectrum. In contrast to the calculations performed within the Born approximation, these coefficients depend on the nuclear charge. For high nuclear charges and low energies the results of our calculation show considerable deviations from the results of the Born approximation.

For the electric monopole conversion we find that the angular distribution obtained with the scattering waves is more anisotropic compared to the Born approximation. In contrast, in the case of the electric and magnetic pair conversion of multipolarity $L>0$, we see that the angular correlation function is not as strongly peaked around the opening angle $\theta=0^{\circ}$. In both cases the maximum of the angular distribution can be shifted from $\theta=0^{\circ}$ to $\theta=180^{\circ}$ for supercritical nuclear charges $Z>172$ which are expected to be generated for a short time in heavy-ion collisions.

The only experimental result which was available to us - the electric monopole transition in ${ }_{8}^{16} \mathrm{O}$ with transition energy $\omega=6 \mathrm{MeV}$ - tends to confirm our result which, however, at this low- $Z$ value agrees with the Born approximation. It is left to future studies on this subject to check the validity of the approximations made in the $E 0$ case by taking into account only $j=\frac{1}{2}$ wave functions. In addition, for the EL and ML conversion, the penetration effects have to be examined in an exact manner as well as the contributions of higher orders in the perturbation expansion.

In recent GSI positron experiments the narrow line structures in coincident electron and positron spectra are measured for several opening angles. Theoretical predictions are now available for the angular correlation in the case of $\mathrm{EL}, \mathrm{ML}$, and $E 0$ conversion. With the knowledge of the angular correlation of dynamically created electrons and positrons, ${ }^{11}$ the processes which determine mainly the background in these measurements are known. This represents an important tool which might help to enlighten the origin of the peak structures.

\section{ACKNOWLEDGMENTS}

We acknowledge many enlightening discussions with $\mathbf{J}$. Augustin and O. Graf. We would especially like to thank B. Müller for very helpful discussions and for carefully reading the manuscript.

\section{APPENDIX A: RADIAL ELECTRON WAVE FUNCTIONS FOR AN EXTENDED NUCLEUS}

In the case of an extended nucleus we have to discriminate between the alternatives $(Z \alpha)^{2}<\kappa^{2}$ and $(Z \alpha)^{2}>\kappa^{2}$. Inside the nucleus in both cases one can use a power series ansatz. ${ }^{24}$ For $\kappa=k>0$ we have

$$
\begin{aligned}
& u_{1}^{(i)}=\left(\frac{r}{R_{n}}\right)^{k+1} \sum_{l=0}^{\infty} a_{l}\left(\frac{r}{R_{n}}\right)^{2 l}, \\
& u_{2}^{(i)}=\left(\frac{r}{R_{n}}\right)^{k} \sum_{l=0}^{\infty} b_{l}\left(\frac{r}{R_{n}}\right)^{2 l} .
\end{aligned}
$$

The expansion coefficients obey the following recursion formula: 


$$
\begin{aligned}
& (2 k+2 l+1) a_{l}=\left[R_{n}(W+m)+\frac{3}{2} Z \alpha\right] b_{l}-\frac{1}{2} Z \alpha b_{l-1}, \\
& \left.2(l+1) b_{l+1}=-R_{n}(W-m)+\frac{3}{2} Z \alpha\right] a_{l}+\frac{1}{2} Z \alpha a_{l-1},
\end{aligned}
$$

with the coefficients $a_{-1}=b_{-1}=0 . \quad a_{0}$ is determined by the normalization. For $\kappa=-k<0$ one has to interchange the roles of $u_{1}$ and $u_{2}$ and to change the signs of $W$ and $Z$.

Outside the nucleus the wave functions obey the Dirac equation for a Coulomb potential. The radial parts can be written as $^{22}$

$$
\begin{aligned}
& u_{1}=\sqrt{|W+m|}\left(\phi+\phi^{*}\right), \\
& u_{2}=i \sqrt{|W-m|}\left(\phi-\phi^{*}\right) .
\end{aligned}
$$

The function $\phi$ is given by

$$
\begin{aligned}
\phi=N x^{-1 / 2} & {\left[\cos \eta e^{i \alpha_{+}} M_{-(i y+1 / 2), \gamma}(x)\right.} \\
& \left.+\sin \eta e^{i \alpha_{-}} M_{-(i y+1 / 2),-\gamma}(x)\right]
\end{aligned}
$$

with the factors

$$
\begin{aligned}
& e^{i \alpha_{+}}=\sqrt{-(\gamma+i y) /(\kappa+i y / E)} e^{-i \pi / 2}, \\
& e^{i \alpha_{-}}=\sqrt{(\gamma-i y) /(\kappa+i y / E)} e^{i \pi / 2} .
\end{aligned}
$$

$M_{\alpha, \beta}(x)$ denotes the Whittaker function. ${ }^{25}$ The asymptotic form of this function yields expressions for the normalization factor $N$ and the phase shift

$$
\begin{aligned}
\delta_{\mathrm{stat}, \kappa}=\arg & \left(\cos \eta e^{i \alpha_{+}} \frac{\Gamma(2 \gamma)}{\Gamma(\gamma+1+i y)}\right. \\
& \left.+\sin \eta e^{i \alpha_{-}} \frac{\Gamma(-2 \gamma+1)}{\Gamma(-\gamma+1+i y)}\right),
\end{aligned}
$$

where we left out the unphysical logarithmic phase shift. $\eta$ is obtained from the matching condition at the nuclear radius

$$
\left.\frac{u_{1}^{(i)}}{u_{2}^{(i)}}\right|_{R}=\left.\frac{u_{1}}{u_{2}}\right|_{R} .
$$

In the case of $(Z \alpha)^{2}>\kappa^{2}$ we have imaginary $\gamma$ and the radial functions read as

$$
\phi=N x^{-1 / 2}\left[e^{i \eta} \sqrt{(-\gamma+i y) /(\kappa+i y / E)} M_{-(i y+1 / 2), \gamma}(x)+e^{\left.-i \eta-\pi|\gamma| \sqrt{(\gamma-i y) /(\kappa+i y / E)} M_{-(i y+1 / 2),-\gamma}(x)\right]} .\right.
$$

The phase shift now becomes

$$
\begin{aligned}
\delta_{\text {stat }, \kappa}= & e^{i \eta} \sqrt{(-\gamma+i y) /(\kappa+i y / E)} \frac{\Gamma(2 \gamma+1)}{\Gamma(\gamma+1+i y)} \\
& +e^{-i \eta-\pi|\gamma| \sqrt{(\gamma-i y) /(\kappa+i y / E)}} \frac{\Gamma(-2 \gamma+1)}{\Gamma(-\gamma+1+i y)}
\end{aligned}
$$

(again the logarithmic phase shift is left out).

\section{APPENDIX B: $\gamma$-EMISSION PROBABILITY}

The transition amplitude which describes the decay of an excited nuclear state by $\gamma$ emission is given by

$$
\begin{aligned}
S_{i f}=2 \pi i e \sqrt{\omega / R_{n}} \int & d \tau_{n}\left\langle f\left|\hat{\mathbf{j}}_{n}\left(\mathbf{r}_{n}\right)\right| i\right\rangle \cdot \mathbf{A}_{L M}^{(\tau)^{*}}\left(\omega \mathbf{r}_{n}\right) \\
& \times \delta\left(\varepsilon_{i}-\varepsilon_{f}-\omega\right)
\end{aligned}
$$

The factor $\sqrt{\omega / R_{n}}$ arises from the normalization of the radiation field $\mathrm{A}$ within a sphere with radius $R_{n} . \varepsilon_{i}$ and $\varepsilon_{f}$ denote the energy of the nucleus in the initial and the final state, respectively, and the $\gamma$ energy $\omega$ must equal the nuclear transition energy. The fields $\mathbf{A}_{L M}^{(\tau)^{*}}\left(\omega \mathbf{r}_{n}\right)$ are those of Eqs. (29) - (30). We introduce the abbreviation

$$
V_{\gamma}^{(\tau)}(L, M)=\int d \tau_{n} \mathbf{j}_{n}\left(\mathbf{r}_{n}\right) \cdot \mathbf{A}_{L M}^{(\tau)^{*}}\left(\omega \mathbf{r}_{n}\right)
$$

for the nuclear matrix element with $\tau=e, m$. Squaring this and summing over the final nuclear states and averaging over the initial ones we get the $\gamma$-emission probability

$$
\begin{aligned}
P_{\gamma}(L, \tau) & =\frac{8 \pi \alpha \omega}{2 J_{i}+1} \sum_{M_{i}, M_{f}, M}\left|V_{\gamma}^{(\tau)}(L, M)\right|^{2} \\
& =\frac{8 \pi \alpha \omega}{2 J_{i}+1}\left|V_{\gamma}^{(\tau)}(L)\right|^{2}
\end{aligned}
$$

The last equality follows by the use of the Wigner-Eckart theorem; $V_{\gamma}^{(\tau)}(L)$ is the reduced nuclear matrix element.

\section{APPENDIX C: THE ANGULAR CORRELATION FUNCTIONS IN BORN APPROXIMATION}

With the abbreviation $\mathbf{q}=\mathbf{p}^{\prime}+\mathbf{p}$ the expressions for the angular correlation derived in Ref. 1 reads, for the electric pair conversion, as

$$
\begin{aligned}
\gamma_{L}(\theta)=\frac{2 \alpha}{\pi(L+1)} \frac{p p^{\prime}}{q} \frac{(q / \omega)^{2 L-1}}{\left(\omega^{2}-q^{2}\right)^{2}}[ & (2 L+1)\left(W W^{\prime}+1-\frac{1}{3} p p^{\prime} \cos \theta\right)+L\left[\frac{q^{2}}{\omega^{2}}-2\right]\left(W W^{\prime}-1+p p^{\prime} \cos \theta\right) \\
& \left.+\frac{1}{3}(L-1) p p^{\prime}\left[3 / q^{2}\left(p+p^{\prime} \cos \theta\right)\left(p^{\prime}+p \cos \theta\right)-\cos \theta\right]\right],
\end{aligned}
$$


and for the magnetic pair conversion

$$
\gamma_{L}(\theta)=\frac{2 \alpha}{\pi} \underset{q}{q} \frac{p^{\prime}}{\left(\omega^{2}-q^{2}\right)^{2}}\left[1+W W^{\prime}-\left(p p^{\prime} / q^{2}\right)\left(p+p^{\prime} \cos \theta\right)\left(p^{\prime}+p \cos \theta\right)\right]
$$

$\gamma_{L}(\theta)$ corresponds to the angular correlation function $d^{2} \beta /(d E d \cos \theta)$ up to a normalization factor since Rose uses the natural unit system $\hbar=c=m=1$, where $m$ is the electron rest mass. Finally, we give the symmetric expression for the $E 0$ anisotropy factor which is easy to derive in Born approximation

$$
\epsilon_{B}=\frac{p p^{\prime}}{E E^{\prime}-m^{2}}
$$

\section{APPENDIX D: CALCULATION OF THE ANGULAR CORRELATION}

Here we want to fill in some steps leading to the angle-dependent pair-conversion coefficient [Eqs. (46) and (47)]. We start with the differential pair-conversion probability Eq. (26), inserting he explicit expressions of the expansion coefficients, Eq. (14), and the abbreviation $\hat{l}=\sqrt{2 l+1}$ :

$$
\begin{aligned}
& \frac{P_{e^{+} e^{-}}}{d E}=\frac{2 \pi}{2 J_{i}+1} \int d \Omega \int d \Omega^{\prime} \sum_{M_{i}, M_{f}} \sum_{L, M, L^{\prime}, M^{\prime}} \sum_{\lambda, \lambda^{\prime} \kappa^{\prime}, \mu^{\prime}, \kappa, \mu} \sum_{k^{\prime}, m^{\prime}, k, m} \frac{1}{(4 \pi)^{2}}\left(\hat{l}^{\prime} \hat{\imath} \hat{r}^{\prime} \hat{r}\right) \\
& \times\left[\begin{array}{ccc}
l^{\prime} & \frac{1}{2} & j^{\prime} \\
0 & \lambda^{\prime} & -\lambda^{\prime}
\end{array}\right]\left[\begin{array}{ccc}
l & \frac{1}{2} & j \\
0 & \lambda & -\lambda
\end{array}\right]\left[\begin{array}{ccc}
r^{\prime} & \frac{1}{2} & s^{\prime} \\
0 & \lambda^{\prime} & -\lambda^{\prime}
\end{array}\right]\left[\begin{array}{ccc}
r & \frac{1}{2} & s \\
0 & \lambda & -\lambda
\end{array}\right) \\
& \times(-)^{\left(1 / 2-l^{\prime}-\lambda^{\prime}\right)+(1 / 2-l-\lambda)+\left(1 / 2-r^{\prime}-\lambda^{\prime}\right)+(1 / 2-r-\lambda)} e^{i\left(\delta^{\prime}{ }^{\prime}-\delta_{\kappa}-\delta^{\prime}{ }^{\prime}+\delta_{k^{\prime}}{ }^{\prime}-l^{\prime}+l+r^{\prime}-r\right.} \\
& \times D_{\mu^{\prime} \lambda^{\prime}}^{\left(j^{\prime}\right)^{*}}\left(\varphi^{\prime}, \vartheta^{\prime}\right) D_{\mu \lambda}^{(j)}(\varphi, \vartheta+\pi) D_{m^{\prime} \lambda^{\prime}}^{\left(s^{\prime}\right)}\left(\varphi^{\prime}, \vartheta^{\prime}\right) D_{m \lambda}^{(s)^{*}}(\varphi, \vartheta+\pi)\left(\hat{j}^{\prime} \hat{j}^{\prime} \hat{s}^{\prime} \hat{s}\right) \\
& \times\left(\begin{array}{ccc}
J_{f} & L & J_{i} \\
-M_{f} & M & M_{i}
\end{array}\right]\left[\begin{array}{ccc}
J_{f} & L^{\prime} & J_{i} \\
-M_{f} & M^{\prime} & M_{i}
\end{array}\right]\left(\begin{array}{ccc}
j & j^{\prime} & L \\
\mu & -\mu^{\prime} & M
\end{array}\right]\left[\begin{array}{ccc}
s & s^{\prime} & L \\
m & -m & M^{\prime}
\end{array}\right) \\
& \times \frac{(4 \pi \alpha \omega)^{2}}{L(L+1)}(-)^{j^{\prime}-\mu^{\prime}+s^{\prime}-m^{\prime}} \boldsymbol{V}_{\gamma}^{(\tau)}(L) \boldsymbol{V}_{\gamma}^{(\tau)}\left(L^{\prime}\right)\left\langle\boldsymbol{\kappa}^{\prime}\left\|\boldsymbol{Y}_{L}\right\| \boldsymbol{\kappa}\right\rangle\left\langle k^{\prime}\left\|\boldsymbol{Y}_{L^{\prime}}\right\| k\right\rangle \boldsymbol{R}_{\kappa k^{\prime}}(L) \boldsymbol{R}_{k k^{\prime}}^{*}\left(L^{\prime}\right) \text {. }
\end{aligned}
$$

The sums over the nuclear magnetic quantum numbers $M_{i}$ and $M_{f}$ can be carried out by the use of the orthogonality of the $3 j$ symbols yielding Kronecker deltas which cancel the sums over $L^{\prime}$ and $M^{\prime}$. We transform the rotation matrices according to

$$
D_{\mu^{\prime} \lambda^{\prime}}^{\left(j^{\prime}\right.}\left(\varphi^{\prime}, \vartheta^{\prime}\right)=(-)^{\mu^{\prime}-\lambda^{\prime}} D_{-\mu^{\prime}-\lambda^{\prime}}^{j^{\prime}}\left(\varphi^{\prime}, \vartheta^{\prime}\right)
$$

and

$$
D_{m \lambda}^{(s)^{*}}(\varphi, \vartheta+\pi)=(-)^{m-\lambda} D_{-m-\lambda}^{s}(\varphi, \vartheta+\pi),
$$

and are now able to combine any two of the rotation matrices with the same arguments to one rotation matrix and two $3 j$ symbols. ${ }^{15}$ We obtain

$$
\begin{aligned}
& \frac{d P_{e^{+} e^{-}}}{d E}=\frac{2 \pi(\alpha \omega)^{2}}{2 J_{i}+1} \sum_{L}\left|V_{\gamma}^{(\tau)}(L)\right|^{2} \int d \Omega \int d \Omega^{\prime} \sum_{\kappa, \kappa^{\prime}, k, k^{\prime}} \sum_{\lambda, \lambda^{\prime}, M} \sum_{\mu, \mu^{\prime}, m, m^{\prime}, \gamma, \gamma, \delta, \epsilon} \sum_{I, J} e^{i\left(\delta_{\kappa^{\prime}}^{\prime}-\delta_{\kappa}-\delta_{k^{\prime}}^{\prime}+\delta_{k}^{\prime}\right)} D_{\beta \gamma}^{(I)}\left(\varphi^{\prime}, \vartheta^{\prime}\right) D_{\delta \epsilon}^{(J)}(\varphi, \vartheta+\pi) \\
& \times(-)^{j^{\prime}+s^{\prime}+l^{\prime}+l+r^{\prime}+r-\mu^{\prime}-m-\lambda-\lambda^{\prime}-\beta-\gamma-\delta-\epsilon_{i} l-r-l^{\prime}-r^{\prime}}\left(\hat{j}^{\prime} \hat{j} \hat{s} \hat{s}^{\prime}\right)\left(\hat{l}^{\prime} \hat{l} \hat{r}^{\prime} \hat{r}\right) \\
& \times \frac{\left\langle\kappa^{\prime}|| Y_{L} \| \kappa\right\rangle\left\langle k^{\prime}\left\|Y_{L}\right\| k\right\rangle}{L(L+1)(2 L+1)} R_{\kappa \kappa^{\prime}}(L) R_{k k^{\prime}}^{*}(L)(2 I+1)(2 J+1) \\
& \times\left[\begin{array}{ccc}
j & j^{\prime} & L \\
\mu & -\mu^{\prime} & M
\end{array}\right]\left[\begin{array}{ccc}
s & s^{\prime} & L \\
m & -m^{\prime} & M
\end{array}\right]\left[\begin{array}{ccc}
j & s & J \\
\mu & -m & -\delta
\end{array}\right]\left[\begin{array}{ccc}
j^{\prime} & s^{\prime} & I \\
-\mu^{\prime} & m^{\prime} & -\beta
\end{array}\right] \\
& \times\left(\begin{array}{ccc}
r & \frac{1}{2} & s \\
0 & \lambda & -\lambda
\end{array}\right]\left[\begin{array}{ccc}
l & \frac{1}{2} & j \\
0 & \lambda & -\lambda
\end{array}\right]\left(\begin{array}{ccc}
j & s & J \\
\lambda & -\lambda & -\epsilon
\end{array}\right) \\
& \times\left[\begin{array}{ccc}
l^{\prime} & \frac{1}{2} & j^{\prime} \\
0 & \lambda^{\prime} & -\lambda^{\prime}
\end{array}\right]\left[\begin{array}{ccc}
r^{\prime} & \frac{1}{2} & s^{\prime} \\
0 & \lambda^{\prime} & -\lambda^{\prime}
\end{array}\right]\left[\begin{array}{ccc}
j^{\prime} & s^{\prime} & I \\
-\lambda^{\prime} & \lambda^{\prime} & -\gamma
\end{array}\right) .
\end{aligned}
$$


The first row of $3 j$ symbols can be written as a $6 j$ symbol. After renaming the indices the second and third row of $3 j$ symbols are transformed into $3 j$ and $6 j$ symbols. Thus we are left with

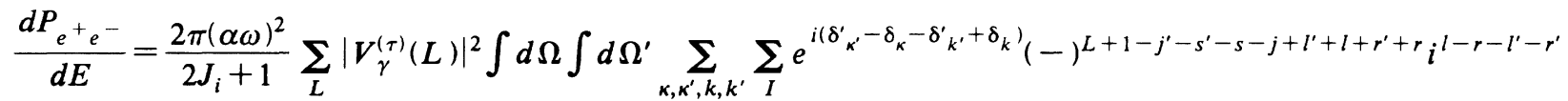

$$
\begin{aligned}
& \times\left(\hat{j}^{\prime} \hat{j} \hat{s}^{\prime} \hat{s}\right)\left(\hat{l}^{\prime} \hat{l}^{\prime} \hat{r}^{\prime} \hat{r}\right) \frac{\left\langle\kappa^{\prime}\left\|Y_{L}\right\| \kappa\right\rangle\left\langle k^{\prime}\left\|Y_{L}\right\| k\right\rangle}{L(L+1)(2 L+1)} R_{\kappa \kappa^{\prime}}(L) R_{k k^{\prime}}^{*}(L)(2 I+1) \\
& \times\left(\begin{array}{lll}
r^{\prime} & l^{\prime} & I \\
0 & 0 & 0
\end{array}\right\}\left\{\begin{array}{lll}
r^{\prime} & l^{\prime} & I \\
j^{\prime} & s^{\prime} & \frac{1}{2}
\end{array}\right\}\left\{\begin{array}{lll}
r & l & I \\
0 & 0 & 0
\end{array}\right\}\left\{\begin{array}{lll}
r & l & I \\
j & s & \frac{1}{2}
\end{array}\right\} \\
& \times \sum_{\beta} D_{\beta 0}^{(I)}\left(\varphi^{\prime}, \vartheta^{\prime}\right) D_{-\beta 0}^{(I)}(\varphi, \vartheta+\pi)\left\{\begin{array}{ccc}
j & I & s \\
s^{\prime} & L & j^{\prime}
\end{array}\right\},
\end{aligned}
$$

where we carried out some summations. The two remaining rotation matrices can be rewritten as a Legendre polynomial which depends now on the angle between the positive-energy and negative-energy electron directions,

$$
\sum_{\beta} D_{\beta 0}^{(I)}\left(\varphi^{\prime}, \vartheta^{\prime}\right) D_{-\beta 0}^{(I)}(\varphi, \vartheta+\pi)(-)^{\beta}=P_{I}(\cos \widetilde{\theta}),
$$

where $\widetilde{\theta}$ is the angle between the lepton directions. We want to introduce the angle between electron and positron direction which is $\theta=\pi-\widetilde{\theta}$ and, therefore, $P_{I}(\cos \widetilde{\theta})=P_{I}(-\cos \theta)=(-)^{I} P_{I}(\cos \theta)$. One angular integral in Eq. (D5) can be carried out since no direction in space is singled out; a polarization of the nucleus is not considered, we average over the nuclear spin projections. The $\varphi$-dependent part of the last integral is carried out as well. The integrations yield a factor of $8 \pi^{2}$.

Inserting the reduced spherical matrix elements, Eq. (40), we get the expression which is referred to as doubly differential pair-conversion probability [Eq. (45)]:

$$
\begin{aligned}
& \frac{d P_{e^{+} e^{-}}}{d E d \cos \theta}=4 \frac{(4 \pi \alpha \omega)^{2}}{2 J_{i}+1} \sum_{L} \frac{\left|V_{\gamma}^{(\tau)}\right|^{2}}{L(L+1)} \sum_{\kappa, \kappa^{\prime}, k, k^{\prime}}\left|\kappa \kappa^{\prime} k k^{\prime}\right| e^{i\left(\delta_{\kappa^{\prime}}^{\prime}-\delta_{\kappa}-\delta^{\prime} k^{\prime}+\delta_{k}\right)}(-)^{L+1} i^{l^{\prime}-r^{\prime}-l+r} R_{\kappa \kappa^{\prime}}(L) R_{k, k^{\prime}}^{*}(L) \\
& \times\left(\begin{array}{ccc}
j & j^{\prime} & L \\
\frac{1}{2} & -\frac{1}{2} & 0
\end{array}\right]\left\{\begin{array}{ccc}
s & s^{\prime} & L \\
\frac{1}{2} & -\frac{1}{2} & 0
\end{array}\right) \sum_{I}(2 I+1) \\
& \times\left[\begin{array}{ccc}
j^{\prime} & s^{\prime} & I \\
\frac{1}{2} & -\frac{1}{2} & 0
\end{array}\right]\left[\begin{array}{ccc}
j & s & I \\
\frac{1}{2} & -\frac{1}{2} & 0
\end{array}\right]\left\{\begin{array}{ccc}
j & I & s \\
s^{\prime} & L & j^{\prime}
\end{array}\right\} P_{I}(\cos \theta)
\end{aligned}
$$

${ }^{1}$ M. E. Rose, Phys. Rev. 76, 678 (1949).

2J. R. Oppenheimer, Phys. Rev. 60, 964 (1941).

${ }^{3}$ M. E. Rose, Relativistic Electron Theory (Wiley, New York, 1961).

${ }^{4}$ H. A. Bethe and L. C. Maximon, Phys. Rev. 93, 768 (1954).

${ }^{5}$ T. A. Green and M. E. Rose, Phys. Rev. 110, 105 (1958).

${ }^{6}$ E. L. Church and J. Weneser, Annu. Rev. Nucl. Sci. 10, 193 (1960); G. vanden Berghe and K. Heyde, Nucl. Phys. A144, 558 (1970).

${ }^{7}$ J. Schweppe, A. Gruppe, K. Bethge, H. Bokemeyer, T. Cowan, H. Folger, J. S. Greenberg, H. Grein, S. Ito, R. Schulé, D. Schwalm, K. E. Stiebing, N. Trautmann, P. Vincent, and M. Waldschmidt, Phys. Rev. Lett. 51, 2261 (1983); M. Clemente, E. Berdermann, P. Kienle, H. Tsertos, W. Wagner, C. Kozhuharov, F. Bosch, and W. Koenig, Phys. Lett. 137B, 41 (1984); T. Cowan, H. Backe, M. Begemann, K. Bethge, H. Bokemeyer, H. Folger, J. S. Greenberg, H. Grein, A. Gruppe, Y. Kido, M. Klüver, D. Schwalm, J. Schweppe, K. E. Stiebing, N. Trautmann, and P. Vincent, Phys. Rev. Lett. 54, 1761 (1985); T. Cowan, in Physics of Strong Fields, Vol. 153 of NATO Advanced Study Institute, Series B: Physics, edited by W. Greiner (Plenum, New York, 1986), p. 111; H. Bokemeyer, ibid., p. 195; W. Koenig, F. Bosch, P. Kienle, C.
Kozhuharov, H. Tsertos, E. Berdermann, S. Huchler, and W. Wagner, Z. Phys. A 328, 129 (1987).

${ }^{8}$ B. Müller, in Atomic Physics of Highly Ionized Atoms, edited by R. Marrus (Plenum, New York, 1989), p. 39; K. Geiger, M. Grabiak, O. Graf, W. Greiner, C. Hofmann, D. C. Ionescu, B. Müller, J. Reinhardt, Th. de Reus, K. Rumrich, A. Schäfer, A. Scherdin, S. Schramm, and G. Soff, in Tests of Fundamental Laws in Physics, Series: Moriond Workshops, edited by $O$. Fackler and J. Tran Thanh Van (Editions Frontièrs, Gif-sur-Yvette, 1989), p. 107; A. Schäfer, J. Phys. G 15, 373 (1989).

${ }^{9}$ P. Schlüter, Th. de Reus, J. Reinhardt, B. Müller, and G. Soff, Z. Phys. A 314, 297 (1983).

${ }^{10}$ E. Berdermann, F. Bosch, P. Kienle, W. Koenig, C. Kozhuharov, H. Tsertos, S. Schuhbeck, S. Huchler, J. Kemmer, and A. Schröter, Nucl. Phys. A488, 683c (1988); P. Kienle, Phys. Scr. T23, 123 (1988); W. Koenig, E. Berdermann, F. Bosch, S. Huchler, P. Kienle, C. Kozhuharov, A. Schröter, S. Schuhbeck, and H. Tsertos, Phys. Lett. B 218, 12 (1989); H. Bokemeyer, P. Salabura, D. Schwalm, and K. E. Stiebing, in Tests of Fundamental Laws in Physics, Series: Moriond Workshops, edited by O. Fackler and J. Tran Thanh Van (Editions Frontièrs, Gif-sur-Yvette, 1989), p. 77. 
${ }^{11}$ O. Graf, J. Reinhardt, B. Müller, W. Greiner, and G. Soff, Phys. Rev. Lett. 61, 2831 (1988).

${ }^{12}$ M. Krämer, B. Blank, E. Bozek, E. Ditzel, E. Kankeleit, G. Klotz-Engmann, C. Müntz, H. Oeschler, M. Rhein, and P. Senger, Phys. Rev. C 40, 1662 (1989).

13J. Augustin, B. Müller, and W. Greiner, Z. Phys. D 14, 317 (1989).

${ }^{14}$ N. F. Mott, Proc. R. London Ser. A 124, 425 (1929).

${ }^{15}$ M. E. Rose, Elementary Theory of Angular Momentum (Wiley, New York, 1957).

${ }^{16}$ M. E. Rose, Multipole Fields (Wiley, New York, 1955).

${ }^{17}$ P. Schlüter, G. Soff, and W. Greiner, Phys. Rep. 75, 327 (1981).

18P. Schlüter, G. Soff, and W. Greiner, Z. Phys. A 286, 149
(1978).

${ }^{19}$ G. Soff, P. Schlüter, and W. Greiner, Z. Phys. A 303, 189 (1981).

${ }^{20}$ P. Schlüter and G. Soff, At. Data Nucl. Data Tables 24, 509 (1979).

${ }^{21}$ P. Schlüter, U. Müller, G. Soff, Th. de Reus, J. Reinhardt, and W. Greiner, Z. Phys. A 323, 139 (1986); P. Schlüter, G. Soff, and W. Greiner, Phys. Rev. C 33, 1816 (1986).

${ }^{22}$ B. Müller, J. Rafelski, and W. Greiner, Nuovo Cimento 18A, 551 (1973).

${ }^{23}$ S. Devons and G. R. Lindsey, Nature 164, 539 (1949).

${ }^{24}$ H.-J. Bär and G. Soff, Physica B\&C 128C, 225 (1985).

${ }^{25}$ M. Abramowitz and I. A. Stegun, Handbook of Mathematical Functions (Dover, New York, 1970). 